\title{
Design of Bessel High Order All Pass Filter Based on AN OTRA
}

\author{
Vivek Bhatt, Sandeep Khantwal, Rahul Negi
}

\begin{abstract}
This paper features a single-input single-output (SISO). The suggested circuit is depend on OTRA which is referred as operational transresistance amplifier. and OTRA recommended on the parameters of its increasing slew rate and large bandwidth as compared to conventional circuit containing operational amplifier. In this paper Bessel Higher order All Pass filter structure are described and their simulation is carried out in PSPICE with the help of $0.5 \mu \mathrm{m}$ CMOS technology and simulation result follows the theoretical approach.
\end{abstract}

Keywords- OTRA, Higher Order All Pass Filter, SISO, Bessel, PSPICE, slew rate

\section{INTRODUCTION}

Operational transresistance amplifier (OTRA) evolved as a solution of current mode analog consisting block. The operational transresistance amplifier is referred as amplifier of current difference or an amplifier of Norton. OTRA has features like high gain current input, voltage output device[1-3]. OTRA has both input and output impedance having low value. Due to internally grounded connections of input terminals of OTRA helps in reducing parasitic capacitances at the input[4-6]. Input resistance is also eliminated due to the grounded connection of input. It gives some merits over the building blocks of analog current mode like operational transconductance amplifier OTA, DVCC[5], (CC) known as current conveyor[6] and CDBA etc. Reduction of the parasitic capacitance at the input terminal which is internally generated is the main factor of an OTRA and produces high slew rate along with wider bandwidth. Several analog filters based on OTRA are represented and reference cited there in all involved large than single OTRA [7]. No papers are described on Bessel second order all pass filter architecture depend on Operational transresistance amplifier [8-10].

The suggested work focuses on to develop a second order All Pass filter structure based on an OTRA that provides Bessel filter response.

Manuscript published on November 30, 2019

* Correspondence Author

Vivek Bhatt*, ECE Department, Tula Institute, Dehradun vivekbhatt1987@gmail.com,_sandeep_kakma@rediffmail.com, rahulnegi815@gmail.com

Sandeep khantwal, ECE Department, Tula Institute, Dehradun vivekbhatt1987@gmail.com, rahulnegi815@gmail.com

Rahul negi, ECE Department, Tula Institute, Dehradun vivekbhatt1987@gmail.com,__sandeep_kakma@rediffmail.com, rahulnegi815@gmail.com

(C) The Authors. Published by Blue Eyes Intelligence Engineering and Sciences Publication (BEIESP). This is an open access article under the CCBY-NC-ND license http://creativecommons.org/licenses/by-nc-nd/4.0/

\section{CiRcuit Description}

The inherent architecture of CMOS depend OTRA is shown in figure number 1[2]. The symbolic representation of an OTRA is given in Figure number 2 and the relative portdependency for an ideal OTRA are summarised as:

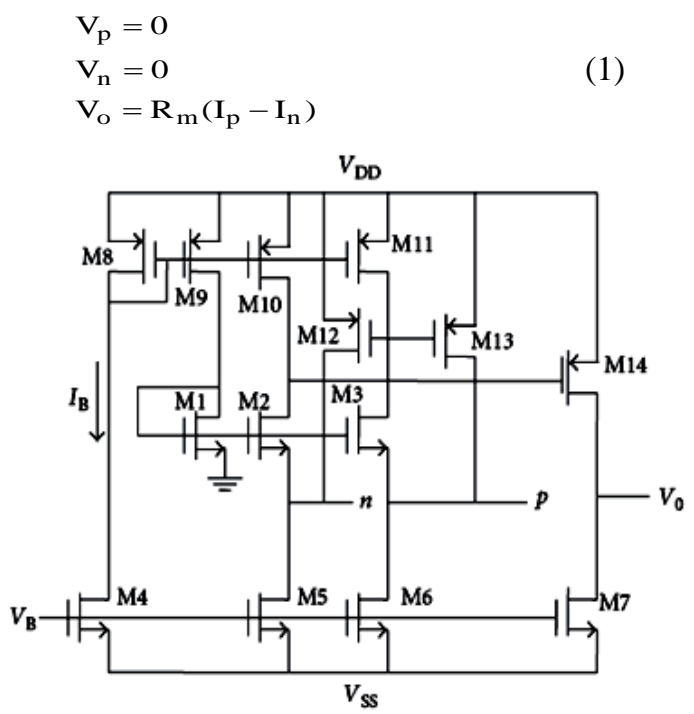

Fig.1. Inherent architecture of OTRA[2]

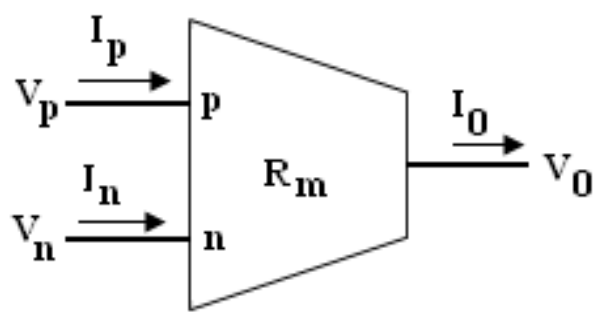

Fig.2. symbolic representation of an OTRA

Where, the $V_{p}$ and $V_{n}$ referred as the input terminals voltage of $p$ and $n$ having a transresistance gain denoted as $R_{m}$, which rises to infinity for the ideal circuit operation. After leaving the magnitude of a signal filter contacts but produces a change in phase. Such type of filter is considered as Al Pass filter. An All-Pass filter which passes all the components of frequency of the input signals without attenuation. However, it gives phase shift which is predictable for different frequencies of the input signals. The response of phase changed from $0^{\circ}$ to $360^{\circ}$ since the frequency is changes from 0 values to infinite. An all pass filter objective is to give equalization of phase in p circuits containing pulse. The transfer function which is generalised of second order All-Pass filter is shown in equation 2:

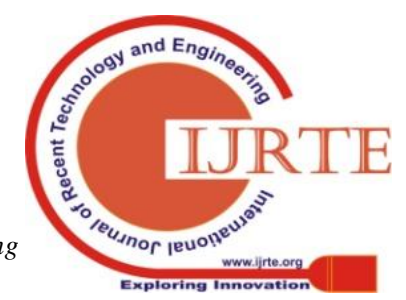




\section{Design of Bessel High Order All Pass Filter Based on AN OTRA}

$$
T(s)=\frac{1-A_{1} s+B_{1} s^{2}}{1+A_{1} s+B_{1} s^{2}}
$$

In which $A_{1}$ and $B_{1}$ are known as the filter coefficient for All-Pass Bessel Filter which value are indicated in Table.1.The architrcture obtained for the OTRA depend second order Bessel Filter are given in figure 3.

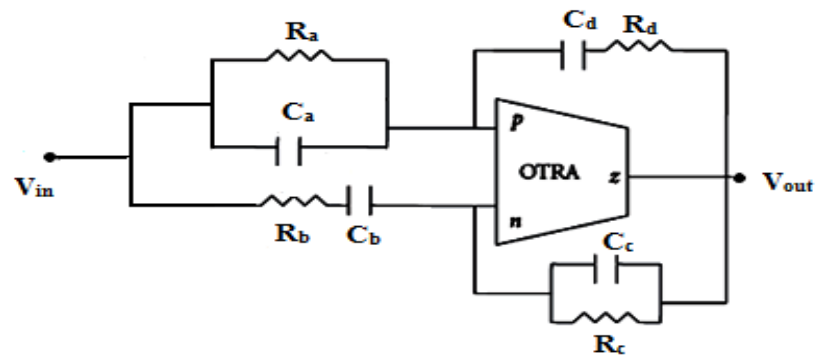

Fig.3. OTRA based second order All Pass filter structure

TABLE.1 SECOND ORDER FILTER COEFFICIENTS

\begin{tabular}{|c|c|}
\hline Name of Filter & Coefficients of filter \\
\hline Bessel Filter & $\mathrm{A}_{1}=1.3617$ \\
& $\mathrm{~B}_{1}=0.6180$ \\
\hline
\end{tabular}

The Bessel filter considered as accurate alternative between two constraints taken as attenuation and phase response. It has feature of no ripple carried out in pass band and moderate flat stop band filter. The transfer function for its RC-RC decomposition methodology [8] for the second order transfer function shown by equation (3)

$$
\mathrm{T}(\mathrm{s})=\frac{\mathrm{N}(\mathrm{s})}{\mathrm{D}(\mathrm{s})}=\frac{\frac{\mathrm{N}(\mathrm{s})}{(\mathrm{s}+1)}}{\frac{\mathrm{D}(\mathrm{s})}{(\mathrm{s}+1)}}
$$

And the routine analysis gives output voltage as

$$
\mathrm{V}_{\text {out }}=\frac{\mathrm{N}(\mathrm{s})}{\mathrm{D}(\mathrm{s})} \mathrm{V}_{\text {in }}
$$

where,

$$
\begin{array}{r}
N(s)=\frac{1}{R_{a} 1}+\frac{C_{a 2} s}{R_{a 2} C_{a 2} s+1}-\frac{C_{b} s}{R_{b} C_{b} s+1} \\
D(s)=\frac{1}{R_{c}}+C_{c} s-\frac{C_{d} s}{R_{d} C_{d} s+1}
\end{array}
$$

The Element Values of filter Passive Components for Bessel Filter is shown in Table. 2

TABLE.2 Element Values of Passive Components.

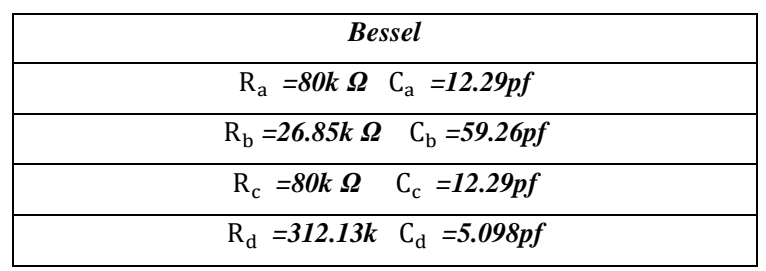

\section{III.SIMULATION RESULTS}

The second order All Pass Bessel filter circuit is evaluated with PSPICE using CMOS depend OTRA and the performance index of the suggested second order All-Pass filters are calculated with the help of CMOS implementation of OTRA having power supply voltages $\mathrm{V}_{s s}=-V_{s S}=1.5$ $\mathrm{Vdc}$ and biasing voltage taken as $\mathrm{V}_{\mathrm{B}}=-0.5 \mathrm{Vdc}$ [2]. The experimental results are introduced through PSPICE depending upon parameters 0.5mCMOS technology. The suggested circuits for varios filters are evaluated for frequency of $100 \mathrm{KHz}$ and for the calculation of the values of the register and capacitor in which ZSF considered as impedance scaling factor $=80 \times 10^{3}$ and FSF considered as frequency scaling factor $(\mathrm{FSF})=2 \pi \times 100 \times 10^{3}$ are taken..In case of the Butterworth All pass second order whose transfer function obtained as (1) the element values of passive components using the admittances are shown in Table. 2 and Figure 4 indicates the simulation results of this circuit. The experimental results satisfy quite well with the theoretical approach.

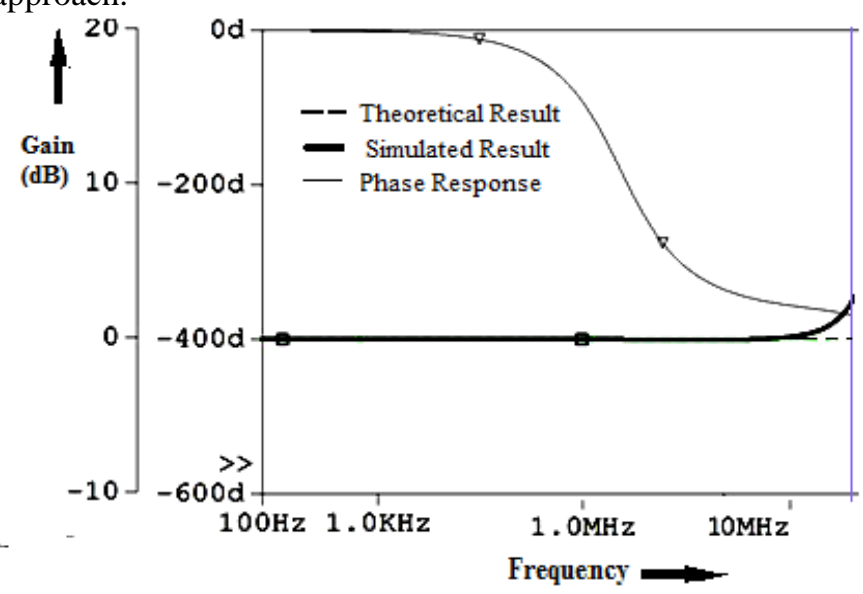

Fig.4.All Pass second order Bessel filter response

\section{IV.CONCLUSION}

In this paper OTRA depend Second Order all-pass Bessel filter structure is described. The suggested filter architecture uses an OTRA along with some passive components. The phase response plot clearly indicates that Bessel filters provides a linear phase response over a wide range of frequency. Theoretical results are proved with the help of simulation in PSPICE depend on parameter of $0.5 \mu \mathrm{m}$ CMOS through MOSIS.

\section{REFERENCES}

1. M. Ghosh, S. K. Paul, R. K. Ranjan, and A. Ranjan, "Third order universal filter using single OTRA", 2013, Journal of EngineeringVolume, 6 pages..

2. H. Mostafa and A. M. Soliman, "A modified CMOS realization of the operational transresistance amplifier (OTRA)", vol. 60, no. 3-4, , 2006, Frequenz , pp. 70-76

3. U. Cam, C. Cakir, O. Cicekoglu, "Novel transimpedance type firstorder all-pass filter using single OTRA", Vol. 58, 2004 AEUInternational Journal of Electronics and Communications, pp. 296298.

4. F. Kacar, "Operational transresistance amplifier based current-mode all-pass filter topologies", 2009. Applied Electronics,pp. 149-152.

5. S. Minaei and E. Yuce, "All-grounded passive elements voltage mode DVCC-based universal filters," vol. 29, no. 2, 2010, Circuits, Systems and Signal Processing, pp. 295-309.

6. J. W. Horng, "High-input impedance voltage-mode universal biquadratic filter using three plus-type CCIIs,", vol. 48, no. 10, 2001, IEEE Transactionson Circuits and Systems II, pp. 996-997.

7. N. Pandey, S. K. Paul, and S. B. Jain, "A new electronically tunable current mode universal filter using MO-CCCII," vol. 58, no. 2, 2009, Analog Integrated Circuits and Signal Processing, pp. 171-178. 
8. K. N. Salama and A. M. Soliman, "Active RC applications of the operational transresistance amplifier," Frequenz, vol. 54, no. 7-8, 2000, Frequenz, pp. 171-176.

9. C. Cakir, U. Cam, and O. Cicekoglu, "Novel allpass filter configuration employing single OTRA," vol. 52, no. 3, 2005, IEEE Transactions onCircuits and Systems II, pp. 122-125.

10. S. kilinc and U. cam, "Cascadable all pass and notch filters employing single operational trans-resistance amplifier," vol. 31, no. 6, 2005, Computers and Electrical Engineering, pp. 391-401.

\section{AUTHORS PROFILE}

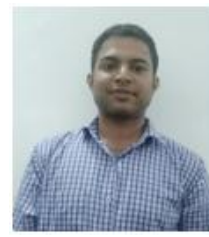

He received his bachelor of technology degree from Arya college of engineering and IT jaipur in 2011 and M.Tech from Bipin Tripathi Kumaon institute of technology Uttarakhand. His research interests include current mode analog circuits. Published 5 papers in international journal.

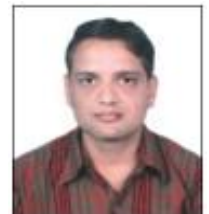

Awarded M.Tech (VLSI DESIGN from Uttarakhand Technical University. B.E. (ECE) from Indira Gandhi government engineering college sagar (Madhya Pradesh). Published 14 paper In International Journal and attended 5 International Conferences. Received Best paper Award titled "Boosted CMOS differential logic and its applications" in INTERNATIONAL CONFERENCE Entitled IDSTM 2018 organized at Ganga technical campus.

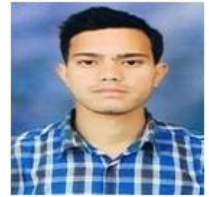

Awarded B.Tech Degree in Applied Electronics and Instrumentation in 2012 from Uttarakhand Technical University and M.Tech degree in Instrumentation and control in 2014 from Graphic Era University. His areas of interests are Biomedical, Instrumentation and Control. 